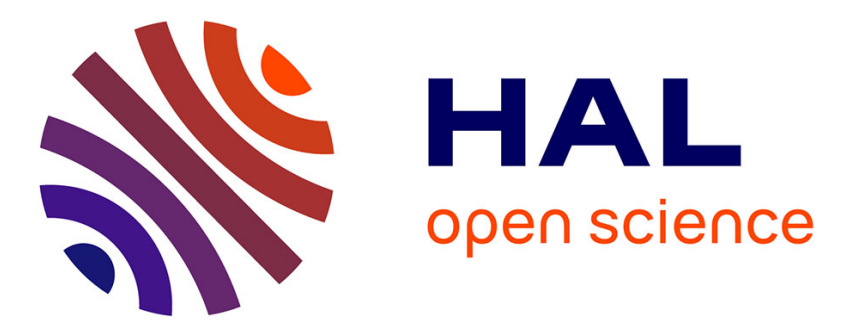

\title{
Multibody kinematics optimization with marker projection improves the accuracy of the humerus rotational kinematics
}

Mickaël Begon, Colombe Belaise, Alexandre Naaim, Arne Lundberg, Laurence Cheze

\section{To cite this version:}

Mickaël Begon, Colombe Belaise, Alexandre Naaim, Arne Lundberg, Laurence Cheze. Multibody kinematics optimization with marker projection improves the accuracy of the humerus rotational kinematics. Journal of Biomechanics, 2017, 62, pp.117-123. 10.1016/j.jbiomech.2016.09.046 . hal01635717

\section{HAL Id: hal-01635717 https://hal.science/hal-01635717}

Submitted on 15 Nov 2017

HAL is a multi-disciplinary open access archive for the deposit and dissemination of scientific research documents, whether they are published or not. The documents may come from teaching and research institutions in France or abroad, or from public or private research centers.
L'archive ouverte pluridisciplinaire HAL, est destinée au dépôt et à la diffusion de documents scientifiques de niveau recherche, publiés ou non, émanant des établissements d'enseignement et de recherche français ou étrangers, des laboratoires publics ou privés. 
Multibody kinematic optimization with marker projection improves the accuracy of the humerus rotational kinematics

Mickaël Begon $^{a}$, Colombe Bélaise $^{a}$, Alexandre Naaim $^{b}$, Arne Lundberg $^{d}$, Laurence Chèze $^{c}$

${ }^{a}$ Department of Kinesiology, Université de Montréal, H3C 3J7, Montreal, QC, CANADA

${ }^{b}$ CIC INSERM 1432, Plateforme d'Investigation Technologique, CHU Dijon, FRANCE

${ }^{c}$ Univ Lyon, Université Lyon 1, IFSTTAR, LBMC UMR_T9406, F69622, Lyon, FRANCE

${ }^{d}$ Karolinska Institutet, Stockholm, SWEDEN

\section{Corresponding author}

Mickaël Begon

Department of Kinesiology, Université de Montréal

Sainte-Justine Hospital Research Center

Address: CEPSUM - 2100, boul. Édouard-Montpetit Bureau 8202

C.P. 6128, succursale Centre-ville

Montréal (Québec) H3C 3J7 CANADA

Phone: 514 343-6151; Fax: 514 343-2181

mickael.begon@umontreal.ca

\section{Original Article}

Word count: 3578 


\section{Abstract}

2 Markers put on the arm undergo large soft tissue artefact (STA). Using markers on the

3 forearm, multibody kinematic optimisation (MKO) helps improve the accuracy of the arm

4 kinematics especially its longitudinal rotation. However deleterious effect of STA may persist

5 and affect other segment estimate. The objective was to present an innovative multibody

6 kinematic optimization algorithm with projection of markers onto a requested axis of the local

7 system of coordinates, to cancel their deleterious effect on this degree-of-freedom. Four subjects

8 equipped with markers put on intracortical pins inserted into the humerus, on skin (scapula, arm

9 and forearm) and subsequently on rigid cuffs (arm and forearm) performed analytic, daily-living,

10 sports and range-of-motion tasks. Scapulohumeral kinematics was estimated using 1) pin markers

11 (reference), 2) single-body optimisation, 3) $\mathrm{MKO}, 4) \mathrm{MKO}$ with projection of all arm markers

12 and 5) MKO with projection of a selection of arm markers. Approaches 2-4 were applied to

13 markers put on the skin and the cuff. The main findings were that multibody kinematic

14 optimization improved the accuracy of 40 to $50 \%$ and the projection algorithm added an extra

$1520 \%$ when applied to cuff markers or a selection of skin markers (all but the medial epicondyle).

16 Therefore, the projection algorithm performed better than multibody and single-body

17 optimizations, especially when using markers put on a cuff. Error of humerus orientation was

18 reduced by half to finally be less than $5^{\circ}$. In conclusion, this innovative algorithm is a promising

19 approach for estimating accurate upper-limb kinematics.

21 Keywords

22 Upper-limb; Multibody kinematic optimization; Marker projection algorithm. 


\section{Introduction}

24 An accurate estimate of the three-dimensional scapulohumeral joint kinematics - especially

25 axial rotation and elevation - can be clinically relevant to assess the rotator cuff muscles

26 disorders (Anglin and Wyss, 2000; Lawrence et al., 2014). Unfortunately, sensors or markers put

27 on the arm undergo soft tissue artefact (STA) (Blache et al., 2016; Hamming et al., 2012) that

28 compromises the accuracy. In particular, the axial rotation tends to be underestimated (Cutti et

29 al., 2005a; Ludewig et al., 2002; Schmidt et al., 1999) and errors up to $30^{\circ}$ on the arm axial

30 rotation were found due to STA, when using an electromagnetic sensor attached to the lateral

31 aspect of the arm (Hamming et al., 2012). Using seven skin markers spread over the arm, this

32 error can be reduced by about $10^{\circ}$ (Begon et al., 2015). However, this last study has shown that

33 single-body optimization with marker weightings fails to improve the arm kinematics, since

34 optimal weightings are subject- and task-specific (Begon et al., 2015). According to the marker-

35 cluster geometrical transformations proposed by Dumas et al. (2014) to describe STA, the

36 movement of a cluster of markers put on the arm with respect to the bone corresponds mainly to a

37 rigid transformation (i.e. translation and rotation) (Blache et al., 2016). Consequently, algorithms

38 using additional markers/sensors on the forearm have been the most promising avenue to

39 accurately estimate the scapulohumeral joint kinematics (Cutti et al., 2005b; Lin and Karduna,

40 2013; Roux et al., 2002; Schmidt et al., 1999). In fact, there is less soft tissue around the forearm

41 and the humeroulnar joint can be modelled as one degree-of-freedom (DoF) in flexion, which

42 decreases the complexity of the multibody system. However, these models have not been

43 validated using the true humeral bone kinematics for a large variety of movements yet, and some

44 algorithms fail when the elbow is fully extended. 
45 In multibody kinematic optimization, markers put on the forearm may correct the arm axial

46 rotation (Roux et al., 2002). Markers put on the olecranon and proximally on the dorsal border of

47 the ulna also may improve the estimation of the arm axial rotation because of the small soft tissue

48 thickness and, above all, because some markers are distant from the arm longitudinal axis when

49 the elbow is bent. The drawback of multibody least-squares minimization is that markers with

50 large STA may negatively affect the kinematics of adjacent segments. For example, some

51 markers on the arm may affect the forearm pro-supination estimation. To the best of our

52 knowledge, no algorithm has been proposed to cancel this deleterious effect. To address this

53 shortcoming, said STA-affected markers could be projected onto an axis of the local frame to

54 ensure that they do not participate to the estimation of the rotation along this axis.

55 The performance of multibody kinematic optimization algorithms depends on the joint 56 constraints (Duprey et al., 2010), model parameters (e.g. segment length (El Habachi et al., 57 2013)) and the markers/sensors involved (Begon et al., 2008). Unfortunately, no optimal marker 58 set or sensor placement exist yet (Anglin and Wyss, 2000). Electromagnetic sensors and cuffs

59 with reflective markers remain the standard in upper-limb biomechanics (Anglin and Wyss, 60 2000). Also, it has been recommended to not attach markers/sensors on anatomical landmarks

61 (Kontaxis et al., 2009). However, recent studies have highlighted that the medial epicondyle

62 presented small STA (Blache et al., 2016) and skin markers performed well (Begon et al., 2015),

63 by comparison with a single electromagnetic sensor (Hamming et al., 2012). Our hypothesis was

64 that skin markers undergo different STA that compensate each other, but this was not confirmed.

65 Since seven markers were put on the arm, it was expected that, in line with simulation-based

66 studies (Challis, 1995; Monnet et al., 2010), the accuracy achieved from them outperformed that 
67 from a single 6-DoF sensor. New multibody kinematic optimization algorithms should then be 68 assessed using different marker sets.

69 The objective of this study was to present an innovative multibody kinematic optimization 70 algorithm, based on the projection of all or selected markers, onto a requested axis of the local 71 system of coordinates (SoC), to cancel their deleterious effect on this DoF. Its benefit was 72 assessed on the upper-limb against true bone kinematics, by comparison to single-body and 73 standard multibody optimizations. 


\section{Methods}

75

76

77

78

79

80

81

82

83 Ltd., Oxford, UK). Fig. 1 illustrates the markers needed for this secondary use of data.

84 Participants performed 18 movements allocated in four categories (Tab. 1): 1) analytic

85 movements similar to Hamming et al. (2012); 2) mimics of activities of daily living (ADL) (van

\subsection{Experiment}

The experimental protocol with pin insertion and skin markers is described in Dal Maso et al. (2015) and Begon et al. (2015). It was approved by the local ethic committees of the Université de Montréal and Karolinska Institutet. Four asymptomatic males (ages: 41, 32, 44 and 27 years, heights: $1.82,1.72,1.77$ and $1.65 \mathrm{~m}$, masses: $80,82,115$ and $57 \mathrm{~kg}$ ) volunteered in this study. Markers were placed on a pin screwed into their humerus and on skin. Subsequently, markers placed on thermoformed cuffs attached to the arm and forearm replaced the skin markers. Marker trajectories were acquired at $300 \mathrm{~Hz}$ using an 18-camera motion analysis system (Oxford Metrics Andel et al., 2008); 3) mimics of sports activities and 4) movements with maximal range of motion (Haering et al., 2014). In the condition with cuffs, only analytic movements and ADL were performed due to the anesthetic duration.

\section{Please insert Fig. 1 here \\ Please insert Fig. 2 here \\ Please insert Tab. 1 here}

\subsection{Models and kinematic reconstruction}

90 For each participant, five multibody kinematic models were developed according to the

91 framework of Kontaxis et al. (2009) and the ISG recommendations (Wu et al., 2005) about

92 anatomical frames definition. They mainly differed in joint and motor constraints. Partially

93 described in Jackson et al. (2012), the models were composed of a common root: thorax 
94 (6 DoFs), clavicle (2 DoFs), scapula (3 DoFs) (Fig. 1). Skin markers were used for these three

95 body segments as proposed by Jackson et al. (2012), including three markers put on the 96 acromion. The joint between the pelvis and thorax was functionally located (Ehrig et al., 2006),

97 while sternoclavicular and acromioclavicular joint locations were defined using markers placed

98 on the most ventral point on the sternoclavicular joint and the most dorsal point on the

99 acromioclavicular joint (Michaud et al., 2016). The generalized coordinates (q) of this common

100 root were first obtained by solving a non-linear least-squares problem as described by

$101 \quad$ Laitenberger et al. (2015).

102 In the $1^{\text {st }}$ and $2^{\text {nd }}$ models (termed as Humerus and Arm models, respectively), the arm had 6

103 DoFs with respect to the scapula; the two models differed in the markers used in the inverse

104 kinematics algorithm: pin markers vs. skin/cuff markers, respectively. The Humerus model

105 corresponded to the reference bone kinematics. The Arm model was about making a single-body

106 optimization. The $3^{\text {rd }}$ to $5^{\text {th }}$ models (UpLimb, UpLimbP and UpLimbS) included the arm (3 DoFs)

107 and forearm (1 DoF) with skin markers. UpLimb model corresponded a standard multibody

108 kinematic optimisation. In the UpLimbP model, all the skin markers of the arm were projected

109 onto its longitudinal axis (y). In the $U p L i m b S$ model, selected skin markers (all of the arm but the

110 medial epicondyle) were projected onto its longitudinal axis. The coordinate systems of the

111 segments and joints were in accordance with the ISG recommendations, based on the same

112 anatomical landmarks for all the models. As suggested by Michaud et al. (2016), the

113 scapulohumeral joint center was determined using the predictive method of Rab et al. (2002). The

114 humeroulnar joint was functionally located (O'Brien et al., 1999). 
115 In all the models, the generalized coordinates $(\mathbf{q})$ of the upper-limb were obtained by solving a

116 non-linear least-squares problem and adapted to include a projection function of observed marker 117 global coordinates $\mathbf{M}_{o b s}^{G}$ onto desired axes, $\mathbf{P}_{o b s}^{G}=p\left(\mathbf{M}_{o b s}^{G}, \mathbf{q}\right)$ :

$$
\min _{\mathbf{q}} \frac{1}{2}\left\|p\left(\mathbf{M}_{o b s}^{G}, \mathbf{q}\right)-f(\mathbf{q})\right\|^{2},
$$

118 where $f(\mathbf{q})$ is the forward kinematic function generated using RBDL (Felis, 2011). In models with

119 projection, the markers local coordinates, but that along the longitudinal axis of the arm, were set 120 at 0 . At each iteration of the nonlinear optimization, observed markers were projected onto the 121 arm longitudinal axis:

$$
\begin{aligned}
& \mathbf{M}_{o b s}^{L}=\mathbf{T}_{G}^{L}(\mathbf{q}) \mathbf{M}_{o b s}^{G} \\
& \mathbf{P}_{o b s}^{L}=\left[\begin{array}{ll}
0 & \mathbf{M}_{o b s(y)}^{L} \\
0 & 1
\end{array}\right]^{\mathrm{T}} \\
& \mathbf{P}_{o b s}^{G}=\mathbf{T}_{L}^{G}(\mathbf{q}) \mathbf{P}_{o b s}^{L} .
\end{aligned}
$$

122 where $\mathbf{T}_{G}^{L}$ are $4 \times 4$ homogenous transformation matrices from the Global $(\mathrm{G})$ to the Local $(\mathrm{L})$

123 frames. For each frame, Eq. [1] was solved using a Levenberg-Marquardt algorithm. The initial

124 guess was the generalized coordinates vector found in the previous frame ( $\mathbf{q}=\mathbf{0}$ in the first frame).

125 At each iteration, the Jacobian matrix of the projection was analytically calculated. The Arm,

126 UpLimb and UpLimbP models were reconstructed using two different marker sets, namely: four

127 markers on each cuff, four skin markers on the arm and forearm, as shown in Fig. 2. The

$128 U p L i m b S$ model was reconstructed with skin markers only.

\section{2.3. Data processing}

130 The variety of movements did not allow to have an angle sequence without passing through

131 gimbal lock positions which makes difficult to reliably maintain continuity in angle time histories

132 (Cutti et al., 2008; Phadke et al., 2011; Senk and Chèze, 2006). To avoid such numerical issues, 
133 the data were post-processed and analyzed as follow. For each model, the scapulohumeral

134 matrices of rotation were calculated using the generalized coordinates. Since high frequency

135 noise may come from multibody kinematic optimization (Fohanno et al., 2014), a zero-lag $2^{\text {nd }}$

136 order Butterworth filter (cuff-off $5 \mathrm{~Hz}$ ) was applied to the time histories of each matrix

137 component, which are continuous. Then, in each frame, the nearest orthonormal matrix (R) was

138 obtained using Horn's algorithm (Horn et al., 1988):

$$
\begin{gathered}
\mathbf{R}=\mathbf{Q}\left(\mathbf{Q}^{\mathrm{T}} \mathbf{Q}\right)^{-1 / 2}, \\
\text { with } \quad\left(\mathbf{Q}^{\mathrm{T}} \mathbf{Q}\right)^{-1 / 2}=\sum_{i=1}^{3} \frac{1}{\sqrt{\lambda_{i}}} e_{i} e_{i}^{\mathrm{T}},
\end{gathered}
$$

139 where $\mathbf{Q}$ is the non-orthonormal matrix after signal processing, $\lambda_{i}$ the eigen values and $e_{i}$ the 140 eigen vectors of $\mathbf{Q}^{\mathrm{T}} \mathbf{Q}$.

141 The scapulohumeral matrix of the Arm, UpLimb and UpLimbP models were compared to that 142 of the Humerus model. The misorientation was characterized by four angular deviations, namely:

143 the total deviation value (Eq. [4a], $\Delta_{t o t}$ ) (Begon et al., 2015; de Vries et al., 2010) and the three 144 axial deviations (Eq. [4b-d]), i.e. the smallest angles between the vectors $x, y$ and $z$ (de Vries et 145 al., 2010) of the Arm, UpLimb, UpLimbP or UpLimbS local frame relative to the local axes of the 146 Humerus model:

$$
\begin{aligned}
& \Delta_{\text {tot }}^{m}=\cos ^{-1}\left(\frac{\operatorname{trace}\left(\mathbf{R}_{\mathbf{1}}^{\mathrm{T}} \mathbf{R}_{\mathbf{m}}\right)-1}{2}\right) \\
& \Delta_{\text {ant }}^{m}=\cos ^{-1}\left(x_{1} \cdot x_{m}\right) \\
& \Delta_{\text {long }}^{m}=\cos ^{-1}\left(y_{1} \cdot y_{m}\right) \\
& \Delta_{\text {lat }}^{m}=\cos ^{-1}\left(z_{1} \cdot z_{m}\right)
\end{aligned}
$$


147 where the 1-index is relative to the Humerus model and $m$-index is relative to the Arm, UpLimb, $148 U p L i m b P$ or $U p L i m b S$ model. These four angles vary between 0 and $180^{\circ}$ and are continuous 149 throughout any movement (i.e. without $\pm 2 \pi$ jumps) by contrast to Euler/Cardan angles.

150 To not artificially underestimate the average misorientation, the static phases at the beginning 151 and end of each movement were removed. Since the number of repetitions was different between 152 subjects - due to discomfort or reduced anesthetic time -, trials of similar movements were 153 concatenated before statistical analysis (Tab. 1). Then, data were reduced to both average and

154 peak values (noted $\bar{\Delta}$ and $\widehat{\Delta}$, respectively) of the total and the axial deviations for each movement 155 and each subject. Results were reported as mean \pm standard deviation. Since all the models have a 156 common root, these deviations corresponded to the humeral misorientation. To test the effect of 157 the model, mean and peak deviations were analyzed using separated repeated measures ANOVAs 158 with Bonferroni-corrected contrasts $(\alpha=0.05)$. Based on our hypotheses, the post-hoc tests were 159 limited to Arm vs. UpLimb, UpLimb vs. UpLimbP and UpLimb vs. UpLimbS $(\alpha=0.05 / 3)$. The 160 gain in accuracy when using a multibody kinematic model and then marker projection was 161 calculated. Finally, 2D correlation coefficients were calculated for time histories of the 162 scapulohumeral unit vectors (lateral, anterior and longitudinal vectors, i.e. the columns of the 163 scapulohumeral rotation matrix) between Arm, UpLimb, UpLimbP or UpLimbS models and the 164 Humerus model to assess their shape agreement. 


\section{Results}

166 The last columns of Tab. 1 summarize the number of frames available in each movement (i.e.

167 a series of trials) for calculating average and peak values of humeral deviations. The movements

168 had between $2103 \pm 810$ and $11269 \pm 7325$ frames, i.e. they last up to 38 seconds. The deviation

169 values that characterized the misorientation in humeral kinematics with respect to the true bone

170 kinematics are summarized in Tab. 2 and illustrated in Fig. S1-S4. When using the Arm model

171 (i.e. single-body optimization), the average total deviation $\bar{\Delta}_{\text {tot }}$ exceeded $10^{\circ}$ with peaks up to

$17221.1^{\circ}$. When using cuff markers, the deviation of the longitudinal axis $\left(\Delta_{\text {long }}\right)$ showed lower

173 values than anterior and lateral deviations. With skin markers, the three axes deviations gave

174 similar values.

175 All the ANOVAs with the models as independent variables were significant (all $p<10^{-4}$ ). Post-

176 hoc tests revealed that there was a systematic gain in accuracy (all $p<10^{-4}$ ) from Arm to UpLimb

177 models, with both cuff and skin markers. The gain ranged between $37 \%\left(\bar{\Delta}_{\text {lat }}\right.$ with skin markers $)$

178 and $63 \%\left(\widehat{\Delta}_{\text {long }}\right.$ with skin markers). Using a multibody model, the total deviation dropped on

179 average to $6.1 \pm 3.2^{\circ}$ and $5.3 \pm 1.9^{\circ}$ for the cuff and skin markers, respectively, with peaks up to

$180 \quad 11.1 \pm 4.8^{\circ}$ for cuff markers but $\leq 10^{\circ}$ when using skin markers.

181 With marker projection on the longitudinal arm axis, the gain relative to a standard multibody

182 kinematic optimization (UpLimb) was different depending on the set of markers (skin vs. cuff)

183 and the model (UpLimbP vs. UpLimbS). When markers were stuck on cuffs, average deviations

184 were significantly reduced $(0.0011<p<0.0023)$ except for $\widehat{\Delta}_{\text {long }}(p=0.3219)$. The gain on the total

185 deviation was $18 \%$. However, the peak values were not significantly different between $U p L i m b P$

186 and UpLimb models (all $p>0.6590$ ). Similar results were found when three skin markers of the

187 arm (all but the medial epicondyle) were projected ( $\operatorname{LpLimbS}$ ): average misorientations were 
188 improved up to $20 \%$ and the peak values were not improved. Using this innovative projection

189 algorithm (UpLimbP with cuff markers and $U p L i m b S)$, the average error in humeral orientation 190 remained below $5^{\circ}$.

191 When all the skin markers of the arm were projected (UpLimbP) onto its longitudinal axis, 192 results were opposite. The average misorientation was not significantly improved (all $p>0.4843$ )

193 and all misorientation peak values were significantly increased (all $p<0.0005$ ), of about $30 \%$, i.e. $1943^{\circ}$.

195 All 2D-correlation coefficients (Table 3) were excellent $(r>0.96)$. They corroborated the 196 results of the ANOVAs since their value increased when using standard multibody kinematic 197 optimization (UpLimb vs Arm) and even more with projection of cuff markers (UpLimbP) and 198 selection of skin markers $(U p L i m b S)$.

Please insert Tab. 2 here

Please insert Tab. 3 here 
An innovative multibody kinematic optimization algorithm with marker projection was developed, validated against true bone kinematics and compared to single-body and multibody optimizations. Its originality relies on the projection of markers onto a chosen anatomical axis to cancel their deleterious effect on the associated rotation due to large STA. Said algorithm was applied to the upper-limb with projection of the arm markers onto the longitudinal axis. The main findings were that multibody optimization improved the accuracy of 40 to $50 \%$ and the projection added an extra $20 \%$ when applied to cuff markers or a selection of skin markers. While only four subjects were involved, the findings relied on 4 categories of movements (analytic, ADL, sports activities and range of motion) including 18 movements from 5 to 36 seconds composed of up to 20910 trials.

\subsection{Accurate humeral kinematics due to multibody optimization}

211 Without multibody optimization, the maximal total deviation exceeded $20^{\circ}$ with larger

212 deviation for axial rotation than flexion and abduction (Fig. S2). These results agree with study of

213 Hamming et al. (2012), also based on pin data. Large deviations were also found in ADL, sports

214 activities, and range of motion, which reinforces the need of improved models for any kind of 215 motion analysis.

216 When forearm markers were involved in the multibody system optimization, the deviation 217 dropped to a few degrees with improved 2D correlation coefficients. This confirms the advantage 218 of multibody over single-body optimization on the upper-limb, mainly highlighted using

219 simulation (Roux et al., 2002). The values of the axis deviations $\left(\Delta_{\text {lat }}, \Delta_{\text {ant }}, \Delta_{\text {long }}\right)$ being similar,

220 the remaining deviation was not associated anymore to the axial rotation. Similar results were 221 found in Lin and Karduna (2013) for three movements exciting one DoF at a time. The present 
222 study generalises the fact that reconstructions including markers on the forearm are efficient to

223 estimate humeral kinematics, based on true bone kinematics and a large variety of movements.

224 The projection of markers onto a desired axis related to a degree-of-freedom is the most

225 innovative feature in this study. In some conditions, it contributed to improve the accuracy to $5^{\circ}$.

226 The remaining deviation may be associated to the estimation of both the elbow flexion axis and

227 the shoulder joint center locations. Similarly to Lin and Karduna (2013), a functional elbow axis

228 was preferred to the midpoint between the medial and lateral epicondyles. The axis obtained from

229 skin/cuff markers did, however, not coincide with the axis given by the pin markers. Also, the

230 scapulohumeral joint location was not chosen as a gold standard position, often unknown in

231 clinical evaluation. An error of about $5 \mathrm{~mm}$ in the scapulohumeral joint location (Michaud et al.,

232 2016) may result in about $5^{\circ}$ as shown for the lower-limb (Stagni et al., 2000). As a perspective,

233 the sensitivity of the method to anatomical axis estimation should be tested. By respecting all the

234 recommendations to accurately define a multibody kinematic model and reconstruct the shoulder

235 kinematics, $5^{\circ}$ may be the floor value when using non-invasive approaches based on

236 optoelectronic systems.

\section{4.2. Algorithm advantages and limitations}

238 The multibody optimization with selected marker projection improved the joint kinematics for

239 a broad range of movements, for four subjects with different anthropometry (IMC from 21 to

$24035 \mathrm{~kg} / \mathrm{m}^{2}$ ). The main advantage of the method is to not have subject- or movement-specific

241 correction, contrary to weighted single-body optimization (Begon et al., 2015) to compensate for

242 the STA of each marker (Blache et al., 2016). However, the selection of markers to be projected

243 remains. 
ADL are generally performed with some elbow flexion (Buckley et al., 1996) but most of the

245 biomechanical evaluation in clinics are composed of arm elevation with extended elbow (Robert-

246 Lachaine et al., 2015). Previous algorithms have to be adjusted when the elbow is extended (Cutti

247 et al., 2006; Schmidt et al., 1999). On the other hand, multibody optimization with or without

248 marker projection is robust to movements where the elbow is extended, such as abduction,

249 flexion (ADL category) and arm elevation with neutral or maximal internal/external arm rotation

250 (RoM category).

251 The effect of marker projection was favorable when using cuff markers or with a selection of

252 skin markers. Indeed, the forearm skin markers were put close to the ulna to minimize the effect

253 of the pro-supination which was not included in the model. However, markers put on the cuff

254 may undergo pro-supination. Similarly to the arm markers, these markers may be projected onto

255 the pro-supination axis of the forearm which should be functionally estimated, as previously

256 suggested by Cutti et al. (2006), since it is not orthonormal to the elbow flexion axis. Further

257 studies are required to assess the algorithm combined with an upper-limb model including pro-

258 supination and hand which may interfere with other DoFs.

259 This algorithm with marker projection may be applied to other segments, especially the thigh

260 and the scapula to offset the deleterious effect of markers with respect to selected DoFs. Since the

261 thigh has a larger diameter and knee flexion varies between 0 and $20^{\circ}$ during the stance phase

262 (Cheze, 2000; Reinschmidt et al., 1997), the projection of the thigh markers onto its longitudinal

263 axis may result in some correction of the thigh rotation. The main limitation would be the

264 estimation of the knee model, which is key in the lower-limb kinematics estimation (Duprey et

265 al., 2010). Regarding the scapula, markers are subject to large STA, except for the acromion and

266 lateral part of the scapula spine (Blache et al., 2016; Matsui et al., 2006). The projection of the 
267 former markers may improve the estimation of the tilt and upward rotation without compromising

268 that of the external rotation. While this algorithm is specific to optoelectronic systems, it could be

269 adapted to electromagnetic/inertial sensors. In fact, the angle associated to the arm rotation

270 should not be introduced into the fusion algorithm (e.g. extended Kalman filter) of the upper-limb

271 multibody system.

272 As in the study of Hamming et al. (2012), the scapulohumeral joint kinematics for all the

273 models was obtained using a common root segment. The error was consequently relative to the

274 humerus only; additional misorientation may come from the scapula. Also, the scapulohumeral

275 joint was modelled as a ball-and-socket joint, while this joint is subject to translations up to

$27612.4 \mathrm{~mm}$ (Dal Maso et al., 2015; Dal Maso et al., 2014). Another drawback of our method was to

277 a priori select four skin markers from the seven available in the experiment. This choice was

278 motivated by three reasons. First, the four markers were selected for comparison to the cuff

279 markers $(n=4)$ and, more broadly, to conditions in clinics. Second, the marker put under the

280 deltoid insertion was close to the pin. This marker has shown small STA (Blache et al., 2016)

281 which may be underestimated due to the closeness of the pin. Unlike recommendations of

282 Kontaxis et al. (2009), lateral and medial epicondyles were used because several clinical studies

283 (Anglin and Wyss, 2000) include these markers and our recent study showed a small deformation

284 energy associated to the medial epicondyle (Blache et al., 2016). Finally, the study emphasis was

285 on the multibody kinematic optimisation; further studies should focus on optimal marker sets.

\section{5. Conclusion}

287 To cancel the deleterious effect of some markers on a selected-DoF kinematic reconstruction,

288 an innovative multibody kinematic optimization algorithm with marker projection was presented,

289 where some markers were projected onto the axis associated to the desired DoFs. This innovative 
290 projection algorithm performed better than multibody and single-body optimizations, especially

291 when using markers put on a cuff. Error of humeral orientation was reduced by half to finally be 292 less than $5^{\circ}$. 


\section{Conflict of interest statement}

294 Authors declare no conflict of interest.

\section{Acknowledgments}

296 Funding for this project was provided by the NSERC Discovery grant (RGPIN-2014-03912). We

297 would like to acknowledge Raphaël for his contribution to the development of the algorithm and

298 the respect of the guidelines of Lundberg et al. (2014). 


\section{References}

300 Anglin, C., Wyss, U.P., 2000. Review of arm motion analyses. Proceedings of the Institution 301 of Mechanical Engineers, Part H: Journal of Engineering in Medicine 214, 541-555.

302 Begon, M., Dal Maso, F., Arndt, A., Monnet, T., 2015. Can optimal marker weightings 303 improve thoracohumeral kinematics accuracy? Journal of Biomechanics, In Press, Corrected 304 Proof

305 Begon, M., Wieber, P.B., Yeadon, M.R., 2008. Kinematics estimation of straddled movements 306 on high bar from a limited number of skin markers using a chain model. Journal of Biomechanics $307 \quad 41,581-586$.

308 Blache, Y., Dumas, R., Lundberg, A., Begon, M., 2016. Main component of soft tissue artefact 309 of the upper-limbs with respect to different arm movements. Journal of Biomechanics (in 310 revision).

311 Buckley, M.A., Yardley, A., Johnson, G.R., Cams, D.A., 1996. Dynamics of the Upper Limb 312 during Performance of the Tasks of Everyday Living - A Review of the Current Knowledge 313 Base. Journal of Engineering in Medicine 210, 241-247.

314 Challis, J.H., 1995. A procedure for determining rigid body transformation parameters. Journal 315 of Biomechanics 28, 733-736.

316 Cheze, L., 2000. Comparison of different calculations of three-dimensional joint kinematics

317 from video-based system data. Journal of Biomechanics 33, 1695-1699.

318 Cutti, A.G., Cappello, A., Davalli, A., 2005a. A new technique for compensatinf the soft tissue 319 artefact at the upper-arm: in vitro validation. Journal of Mechanics in Medicine and Biology 5, 132015. 

compensates for soft tissue artefact in the upper-arm: Preliminary results. Clinical Biomechanics 21, S13-S19.

324 Cutti, A.G., Giovanardi, A., Rocchi, L., Davalli, A., Sacchetti, R., 2008. Ambulatory 325 measurement of shoulder and elbow kinematics through inertial and magnetic sensors. Medical \& 326 Biological Engineering \& Computing 46, 169-178.

327 Cutti, A.G., Paolini, G., Troncossi, M., Cappello, A., Davalli, A., 2005b. Soft tissue artefact 328 assessment in humeral axial rotation. Gait \& Posture 21, 341-349.

329 Dal Maso, F., Raison, M., Lundberg, A., Arndt, A., Allard, P., Begon, M., 2015. 330 Glenohumeral translations during range-of-motion movements, activities of daily living, and 331 sports activities in healthy participants. Clinical Biomechanics 30, 1002-1007.

332 Dal Maso, F., Raison, M., Lundberg, A., Arndt, A., Begon, M., 2014. Coupling between 3D 333 displacements and rotations at the glenohumeral joint during dynamic tasks in healthy 334 participants. Clinical Biomechanics 29, 1048-1055.

335 de Vries, W.H.K., Veeger, H.E.J., Cutti, A.G., Baten, C., van der Helm, F.C.T., 2010.

336 Functionally interpretable local coordinate systems for the upper extremity using inertial \& 337 magnetic measurement systems. Journal of Biomechanics 43, 1983-1988.

338 Dumas, R., Camomilla, V., Bonci, T., Cheze, L., Cappozzo, A., 2014. Generalized 339 mathematical representation of the soft tissue artefact. J Biomech 47, 476-481.

340 Duprey, S., Cheze, L., Dumas, R., 2010. Influence of joint constraints on lower limb 341 kinematics estimation from skin markers using global optimization. Journal of Biomechanics 43, $342 \quad 2858-2862$.

343 Ehrig, R.M., Taylor, W.R., Duda, G.N., Heller, M.O., 2006. A survey of formal methods for 344 determining the centre of rotation of ball joints. Journal of Biomechanics 39, 2798-2809. 
El Habachi, A., Duprey, S., Cheze, L., Dumas, R., 2013. Global sensitivity analysis of the

346 kinematics obtained with a multi-body optimisation using a parallel mechanism of the shoulder.

347 Comput Method Biomec 16, 61-62.

348 Felis, M.L., 2011. RBDL - Rigid Body Dynamics Library : http://rbdl.bitbucket.org/.

349 Fohanno, V., Begon, M., Lacouture, P., Colloud, F., 2014. Estimating joint kinematics of a

350 whole body chain model with closed-loop constraints. Multibody System Dynamics 31, 433-449.

351 Haering, D., Raison, M., Begon, M., 2014. Measurement and description of three-dimensional

352 shoulder range of motion with degrees of freedom interactions. Journal of biomechanical

353 engineering 136, 084502.

354 Hamming, D., Braman, J.P., Phadke, V., LaPrade, R.F., Ludewig, P.M., 2012. The accuracy of

355 measuring glenohumeral motion with a surface humeral cuff. Journal of Biomechanics 45, 1161-

3561168.

357 Horn, B.K.P., Hilden, H.M., Negahdaripour, S., 1988. Closed-form solution of absolute 358 orientation using orthonormal matrices. Journal of the Optical Society of America A 5, 11273591135.

360 Jackson, M., Benkhemis, I., Begon, M., Sardain, P., Vallée, C., Lacouture, P., 2012.

361 Identifying the criterion spontaneously minimized during the take-off phase of a sub-maximal

362 long jump through optimal synthesis. Multibody System Dynamics 28, 225-237.

363 Kontaxis, A., Cutti, A.G., Johnson, G.R., Veeger, H.E.J., 2009. A framework for the definition 364 of standardized protocols for measuring upper-extremity kinematics. Clinical Biomechanics 24, $365246-253$.

366 Laitenberger, M., Raison, M., Perie, D., Begon, M., 2015. Refinement of the upper limb joint 367 kinematics and dynamics using a subject-specific closed-loop forearm model. Multibody System 368 Dynamics 33, 413-438. 

of 3-dimensional shoulder complex kinematics in individuals with and without shoulder pain,

371 part 2: glenohumeral joint. journal of orthopaedic \& sports physical therapy 44, 646-B643.

372 Lin, Y.-L., Karduna, A.R., 2013. Sensors on the Humerus Are Not Necessary for an Accurate 373 Assessment of Humeral Kinematics in Constrained Movements. Journal of Applied 374 Biomechanics 29, 496-500.

375 Ludewig, P.A., Cook, T.M., Shields, R.K., 2002. Comparison of surface sensor and bone-fixed 376 measurement of humeral motion. Journal of Applied Biomechanics 18, 163-170.

377 Lundberg, A., Aguilera, A., Cappozzo, A., Arndt, A., Begon, M., 2014. Entropy in the List of 378 Authors of Scientific Papers. Annals of improbable research 20, 15-17.

379 Matsui, K., Shimada, K., Andrew, P.D., 2006. Deviation of skin marker from bone target 380 during movement of the scapula. Journal of Orthopaedic Science 11, 180-184.

381 Michaud, B., Jackson, M., Arndt, A., Lundberg, A., Begon, M., 2016. Determining in vivo 382 sternoclavicular, acromioclavicular and glenohumeral joint centre locations from skin markers, 383 CT-scans and intracortical pins: A comparison study. Medical engineering \& physics 38, 290384296.

385 Monnet, T., Begon, M., Vallée, C., Lacouture, P., 2010. Improvement of the input data in 386 biomechanics: kinematic and body segment inertial parameters, in: Jerrod H. Levy (Ed.), 387 Biomechanics: Principles, Trends and Applications. Nova Science Publishers, Inc., pp. 353-385.

388 O'Brien, J.F., Bodenheimer Jr, R.E., Brostow, G.J., Hodgins, J.K., 1999. Automatic joint 389 parameter estimation from magnetic motion capture data. Georgia Institute of Technology.

390 Phadke, V., Braman, J.P., LaPrade, R.F., Ludewig, P.M., 2011. Comparison of glenohumeral 391 motion using different rotation sequences. Journal of Biomechanics 44, 700-705. 
Rab, G., Petuskey, K., Bagley, A., 2002. A method for determination of upper extremity 393 kinematics. Gait \& Posture 15, 113-119.

394 Reinschmidt, C., van den Bogert, A.J., Lundberg, A., Nigg, B.M., Murphy, N., Stacoff, A., 395 Stano, A., 1997. Tibiofemoral and tibiocalcaneal motion during walking: external vs. skeletal 396 markers. Gait \& Posture 6, 98-109.

397 Robert-Lachaine, X., Allard, P., Gobout, V., Begon, M., 2015. Shoulder Coordination During 398 Full-Can and Empty-Can Rehabilitation Exercises. Journal of Athletic Training 50, 1117-1125.

399 Roux, E., Bouilland, S., Godillon-Maquinghen, A.P., Bouttens, D., 2002. Evaluation of the 400 global optimisation method within the upper limb kinematics analysis. Journal of Biomechanics $401 \quad 35,1279-1283$.

402 Schmidt, R., Disselhorst-Klug, C., Silny, J., Rau, G., 1999. A marker-based measurement 403 procedure for unconstrained wrist and elbow motions. Journal of Biomechanics 32, 615-621.

404 Senk, M., Chèze, L., 2006. Rotation sequence as an important factor in shoulder kinematics. 405 Clinical Biomechanics 21, S3-S8.

406 Stagni, R., Leardini, A., Cappozzo, A., Grazia Benedetti, M., Cappello, A., 2000. Effects of 407 hip joint centre mislocation on gait analysis results. Journal of Biomechanics 33, 1479-1487.

408 van Andel, C.J., Wolterbeek, N., Doorenbosch, C.A., Veeger, D.H., Harlaar, J., 2008. 409 Complete 3D kinematics of upper extremity functional tasks. Gait \& Posture 27, 120-127.

410 Wu, G., van der Helm, F.C.T., Veeger, H.E.J., Makhsous, M., Van Roy, P., Anglin, C., 411 Nagels, J., Karduna, A.R., McQuade, K., Wang, X.G., Werner, F.W., Buchholz, B., 2005. ISB 412 recommendation on definitions of joint coordinate systems of various joints for the reporting of 413 human joint motion - Part II: shoulder, elbow, wrist and hand. Journal of Biomechanics 38, 981414992. 


\section{Figures and Tables}
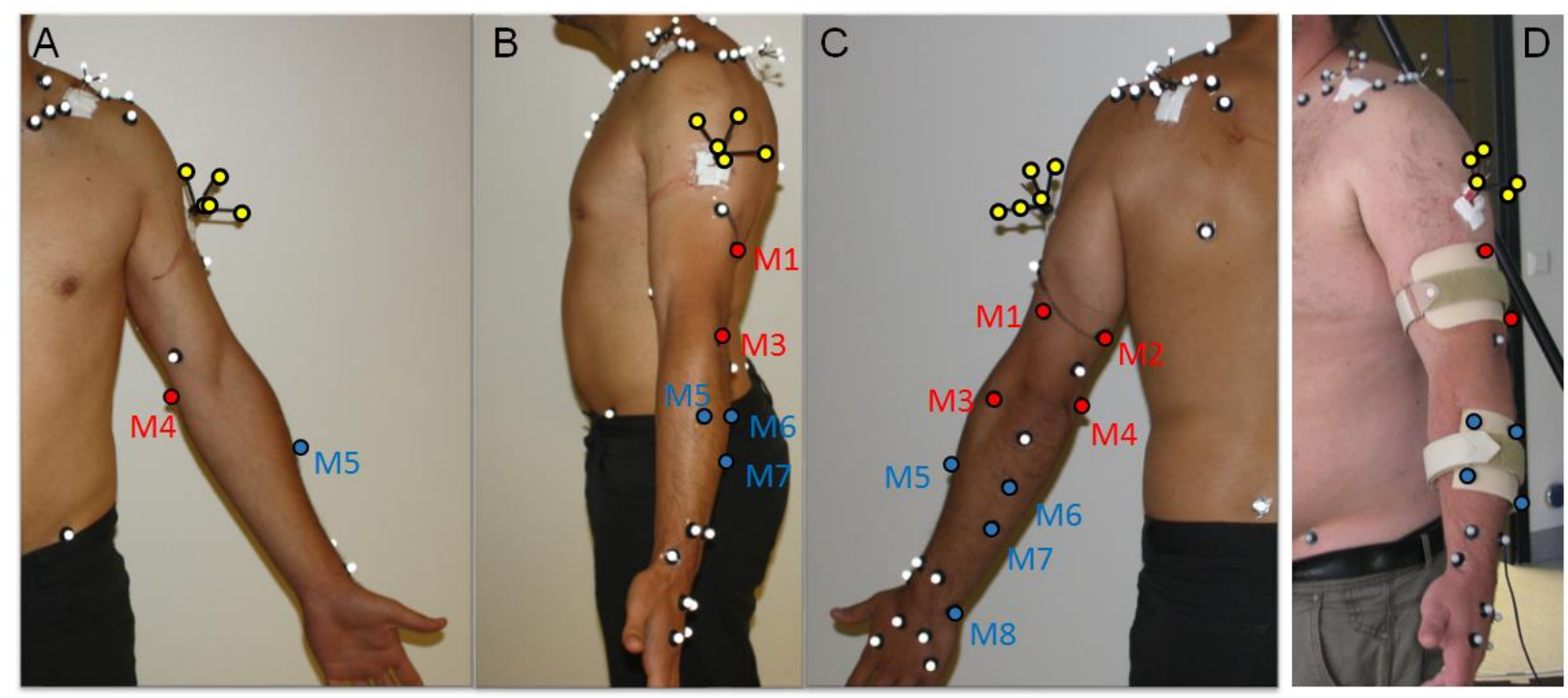

Fig. 1: (A-C) Markers were put on an intracortical pin (in yellow), on the skin of the arm (red) and forearm (blue) and (D) on the cuffs. Specifically, the skin markers were placed as follow: laterally (M1) and medially (M2) to the belly of the triceps, lateral epicondyle (M3), medial epicondyle (M4), medial (M5) and lateral (M6) aspects of the brachioradialis, at the end of the triangle formed by the brachioradialis (M7) and on the ulnar styloid (M8). 


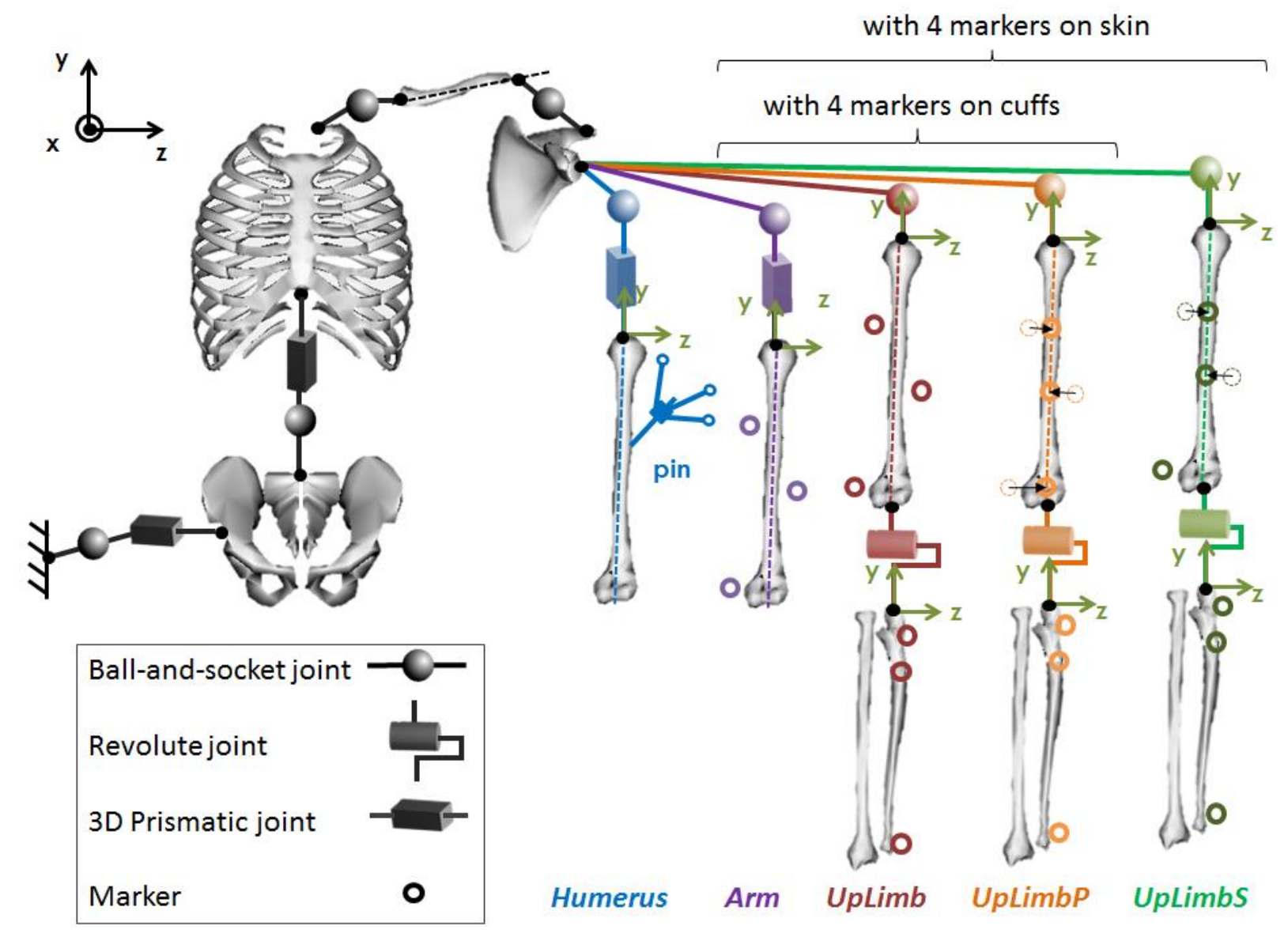

Fig. 2: Five models with the common root (pelvis-thorax-clavicle-scapula) and their degrees-offreedom, from left to right: Humerus with $6 \mathrm{DoFs}$ and based on the markers attached to the intracortical pin screw in the humerus; Arm with 6 DoFs and based on the skin or cuff markers; UpLimb with 3 DoFs at the scapulohumeral joint and 1 DoF at the elbow based on skin and cuff markers; $U p L i m b P$ similar to $U p L i m b$ but all markers on the arm (skin or cuff) are projected onto the longitudinal axis; UpLimbS similar to UpLimb but three markers on the arm (M1-3, not M4) are projected onto the longitudinal axis. 
Tab. 1: Description of the four categories of movements. The last two columns indicate the number of frames retained (mean \pm SD) for the analysis using trials with markers put on the skin or fixed on the cuffs (sampling rate: $300 \mathrm{~Hz}$ ).

\begin{tabular}{|c|c|c|c|c|}
\hline \multirow[t]{2}{*}{ Categories } & \multirow[t]{2}{*}{ Movements } & \multirow[t]{2}{*}{ Comments } & \multicolumn{2}{|c|}{ Number of Frames } \\
\hline & & & Skin & Cuff \\
\hline \multirow{4}{*}{ Analytic movements } & 1. Abduction & up to 10 repetitions & $11188 \pm 6548$ & $9067 \pm 6158$ \\
\hline & 2. Flexion & up to 10 repetitions & $11269 \pm 7325$ & $9550 \pm 6240$ \\
\hline & $\begin{array}{l}\text { 3. Internal-External rotations } \\
\text { with adducted arm }\end{array}$ & & $3806 \pm 1750$ & $4042 \pm 1165$ \\
\hline & $\begin{array}{l}\text { 4. Internal-External rotations with } 90^{\circ} \text { of arm } \\
\text { elevation in the scapular plan }\end{array}$ & & $2782 \pm 1572$ & $3464 \pm 582$ \\
\hline \multirow{5}{*}{$\begin{array}{l}\text { Activities of daily living } \\
\text { (mimics of) }\end{array}$} & 5. Eat** & with a spoon but no food & $2988 \pm 866$ & $2449 \pm 603$ \\
\hline & 6. Comb & with a comb & $4607 \pm 1437$ & $3851 \pm 1251$ \\
\hline & 7. Attach a bra in the back $* *$ & reach back & $3394 \pm 989$ & $3089 \pm 1321$ \\
\hline & 8. Wash armpit**** & reach opposite armpit & $1987 \pm 870$ & $2103 \pm 810$ \\
\hline & 9. Reach front and back pockets & ipsolateral pocket & $5979 \pm 3358$ & $4899 \pm 2782$ \\
\hline \multirow{6}{*}{$\begin{array}{l}\text { Sports activities } \\
\text { (mimics of) }\end{array}$} & 10. Tennis forehands and backhands & with a racket but no ball & $4355 \pm 1042$ & N/A \\
\hline & 11. Throw a ball & without ball & $3243 \pm 1139$ & $\mathrm{~N} / \mathrm{A}$ \\
\hline & 12. Clap & & $1547 \pm 520$ & N/A \\
\hline & 13. Punch* & in a piece of foam* & $3088 \pm 988$ & $\mathrm{~N} / \mathrm{A}$ \\
\hline & & with a stick but no puck & $3962 \pm 130$ & N/A \\
\hline & 14. Hockey shoot & & & \\
\hline \multirow[t]{4}{*}{$\begin{array}{l}\text { Movements with } \\
\text { maximal range of motion }\end{array}$} & $\begin{array}{l}\text { 15. Maximal arm elevation with the arm } \\
\text { maximally internally rotated }\end{array}$ & $\begin{array}{l}\text { in adduction, flexion, abduction and } \\
\text { extension }\end{array}$ & $4823 \pm 1757$ & N/A \\
\hline & $\begin{array}{l}\text { 16. Maximal arm elevation with the arm } \\
\text { maximally externally rotated }\end{array}$ & $\begin{array}{l}\text { in adduction, flexion, abduction and } \\
\text { extension }\end{array}$ & $5511 \pm 1058$ & N/A \\
\hline & $\begin{array}{l}\text { 17. Maximal arm elevation with the arm in neutral } \\
\text { rotation }\end{array}$ & $\begin{array}{l}\text { in adduction, flexion, abduction and } \\
\text { extension }\end{array}$ & $4110 \pm 1516$ & N/A \\
\hline & 18. Maximal internal-external rotation & $\begin{array}{l}\text { for each combination of adduction, } \\
\text { flexion, abduction and extension at } \\
0^{\circ}, 45^{\circ}, 90^{\circ}, 135^{\circ} \text { and } 180^{\circ}\end{array}$ & $9833 \pm 3827$ & N/A \\
\hline
\end{tabular}

* This movement was not performed by subject 1 with skin markers.

** This movement was not performed by subject 3 with cuffs.

N/A not applicable: this movement was not performed with cuff. 
Tab. 2: Mean and standard deviation (SD) in degrees $\left(^{\circ}\right)$ of the four deviations (average $\bar{\Delta}$ and peak $\widehat{\Delta}$ values) for models $A r m$, UpLimb, UpLimbP and UpLimbS relative to Humerus. P-values of the ANOVA and post-hoc tests are in bold when significant and the gain in accuracy (UpLimb vs Arm, UpLimbP vs UpLimb and UpLimbS vs UpLimb) is provided. Values are summarized for two marker sets, namely A) four cuff markers, B) four skin markers on the arm and forearm.

A) Cuff markers ( $\mathrm{n}=35,9$ movements $\mathrm{x} 4$ participants, except for subject 3 who performed 8 movements)

\begin{tabular}{|c|c|c|c|c|c|c|c|c|c|c|c|}
\hline & \multicolumn{2}{|c|}{ Arm } & \multicolumn{3}{|c|}{ UpLimb } & \multicolumn{3}{|c|}{ UpLimbP } & \multicolumn{3}{|c|}{$p$-values } \\
\hline & & & & & & & & & & UpLimb & \\
\hline & Mean & $(\mathrm{SD})$ & Mean & $(\mathrm{SD})$ & Gain & Mean & $(\mathrm{SD})$ & Gain & ANOVA & vs $A r m$ & $U p L i m b$ vs $U p L i m b P$ \\
\hline $\bar{\Delta}_{t o t}$ & 10.5 & $(5.4)$ & 6.1 & $(3.2)$ & $42 \%$ & 5.0 & $(2.3)$ & $18 \%$ & 0.0000 & 0.0000 & 0.0023 \\
\hline $\bar{\Delta}_{\text {lat }}$ & 9.4 & $(5.4)$ & 5.4 & $(3.2)$ & $42 \%$ & 4.2 & $(2.1)$ & $22 \%$ & 0.0000 & 0.0000 & 0.0017 \\
\hline $\bar{\Delta}_{\text {ant }}$ & 9.4 & $(5.0)$ & 5.3 & $(2.7)$ & $43 \%$ & 4.1 & $(2.0)$ & $22 \%$ & 0.0000 & 0.0000 & 0.0011 \\
\hline $\bar{\Delta}_{\text {long }}$ & 5.5 & $(3.3)$ & 3.5 & $(2.4)$ & $36 \%$ & 3.3 & $(2.0)$ & $5 \%$ & 0.0000 & 0.0000 & 0.3219 \\
\hline$\widehat{\Delta}_{t o t}$ & 19.1 & $(8.7)$ & 11.1 & $(4.8)$ & $42 \%$ & 10.9 & $(5.2)$ & $2 \%$ & 0.0000 & 0.0000 & 0.8067 \\
\hline$\hat{\Delta}_{\text {lat }}$ & 17.8 & $(8.2)$ & 9.9 & $(4.6)$ & $44 \%$ & 9.7 & $(4.6)$ & $3 \%$ & 0.0000 & 0.0000 & 0.7112 \\
\hline$\widehat{\Delta}_{\text {ant }}$ & 17.2 & $(7.8)$ & 10.2 & $(4.6)$ & $41 \%$ & 9.9 & $(5.1)$ & $3 \%$ & 0.0000 & 0.0000 & 0.6590 \\
\hline$\widehat{\Delta}_{\text {long }}$ & 12.1 & $(6.0)$ & 7.4 & $(4.3)$ & $39 \%$ & 7.5 & $(4.2)$ & $-2 \%$ & 0.0000 & 0.0000 & 0.6970 \\
\hline
\end{tabular}

B) Skin markers ( $\mathrm{n}=70,18$ movements $\mathrm{x} 4$ participants, except for subject 1 who performed 16 movements)

\begin{tabular}{|c|c|c|c|c|c|c|c|c|c|c|c|c|c|c|c|}
\hline & \multicolumn{2}{|c|}{ Arm } & \multicolumn{3}{|c|}{ UpLimb } & \multicolumn{3}{|c|}{$U p \operatorname{LimbP}$} & \multicolumn{3}{|c|}{ UpLimbS } & \multicolumn{4}{|c|}{$p$-values } \\
\hline & Mean & $(\mathrm{SD})$ & Mean & (SD) & Gain & Mean & $(\mathrm{SD})$ & Gain & Mean & $(\mathrm{SD})$ & Gain & ANOVA & UpLimb vs Arm & $U p L i m b P$ vs $U p L i m b$ & $U p L i m b S$ vs $U p L i m b$ \\
\hline $\bar{\Delta}_{t o t}$ & 10.2 & $(5.3)$ & 5.3 & $(1.9)$ & $48 \%$ & 5.3 & $(1.8)$ & $0 \%$ & 4.4 & $(1.5)$ & $16 \%$ & 0.0000 & 0.0000 & 0.9835 & $\mathbf{0 . 0 0 2 0}$ \\
\hline $\bar{\Delta}_{\text {lat }}$ & 6.8 & $(4.0)$ & 4.3 & $(1.7)$ & $37 \%$ & 4.1 & $(1.7)$ & $4 \%$ & 3.4 & $(1.4)$ & $20 \%$ & 0.0000 & 0.0000 & 0.4843 & 0.0010 \\
\hline $\bar{\Delta}_{a n t}$ & 8.4 & $(4.5)$ & 4.7 & $(1.8)$ & $44 \%$ & 4.7 & $(1.7)$ & $-1 \%$ & 3.9 & (1.4) & $17 \%$ & 0.0000 & 0.0000 & 0.9144 & 0.0023 \\
\hline $\bar{\Delta}_{\text {long }}$ & 8.7 & $(4.8)$ & 3.6 & $(1.5)$ & $59 \%$ & 3.6 & $(1.3)$ & $-1 \%$ & 3.0 & $(1.1)$ & $14 \%$ & 0.0000 & 0.0000 & 0.8370 & 0.0017 \\
\hline$\hat{\Delta}_{t o t}$ & 21.1 & (12.2) & 9.6 & (3.9) & $54 \%$ & 12.4 & $(4.9)$ & $-29 \%$ & 9.3 & $(3.7)$ & $4 \%$ & 0.0000 & 0.0000 & 0.0000 & 0.5362 \\
\hline$\hat{\Delta}_{\text {lat }}$ & 17.0 & $(10.5)$ & 8.5 & $(3.2)$ & $50 \%$ & 11.2 & $(4.7)$ & $-32 \%$ & 8.2 & $(3.6)$ & $3 \%$ & 0.0000 & 0.0000 & 0.0000 & 0.6368 \\
\hline$\hat{\Delta}_{a n t}$ & 17.9 & $(9.7)$ & 8.9 & $(3.7)$ & $\mathbf{5 0 \%}$ & 11.6 & $(4.7)$ & $-30 \%$ & 8.5 & $(3.4)$ & $5 \%$ & 0.0000 & 0.0000 & 0.0001 & 0.4757 \\
\hline$\widehat{\Delta}_{\text {long }}$ & 18.7 & $(11.7)$ & 6.8 & $(3.2)$ & $63 \%$ & 8.5 & $(3.9)$ & $-25 \%$ & 6.8 & $(3.0)$ & $1 \%$ & 0.0000 & 0.0000 & 0.0005 & 0.9295 \\
\hline
\end{tabular}


Tab. 3: Mean and standard deviation (SD) of the $2 \mathrm{D}$ correlation coefficients of lateral $\left(r_{\text {lat }}\right)$, anterior $\left(r_{\text {ant }}\right)$ and longitudinal $\left(r_{\text {long }}\right)$ axes of the Arm, UpLimb, UpLimbP and UpLimbS models and the Humerus model. Values are summarized for two marker sets, namely A) four cuff markers, B) four skin markers on the arm and forearm.

A) Cuff markers ( $\mathrm{n}=35,9$ movements $\mathrm{x} 4$ participants, except for subject 3 who performed 8 movements)

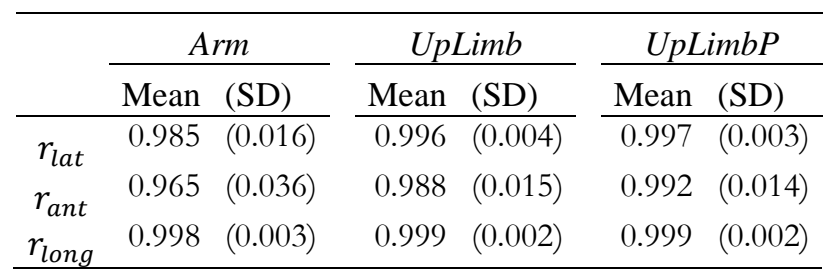

B) Skin markers ( $\mathrm{n}=70,18$ movements $\mathrm{x} 4$ participants, except for subject 1 who performed 16 movements)

\begin{tabular}{|c|c|c|c|c|c|c|c|c|}
\hline & \multicolumn{2}{|c|}{ Arm } & \multicolumn{2}{|c|}{ UpLimb } & \multicolumn{2}{|c|}{ UpLimbP } & \multicolumn{2}{|c|}{ UpLimbS } \\
\hline & Mean & (SD) & Mean & (SD) & Mean & (SD) & Mean & (SD) \\
\hline$r_{l \text { lat }}$ & 0.978 & $(0.115)$ & 0.998 & $(0.002)$ & 0.998 & $(0.002)$ & 0.998 & $(0.002)$ \\
\hline$r_{\text {ant }}$ & 0.975 & $(0.074)$ & 0.994 & $(0.005)$ & 0.994 & $(0.005)$ & 0.996 & $(0.005)$ \\
\hline$r_{\text {long }}$ & 0.981 & $(0.023)$ & 0.998 & $(0.002)$ & 0.998 & $(0.002)$ & 0.998 & $(0.002)$ \\
\hline
\end{tabular}

